\title{
Autonomous CPSoS for cognitive large manufacturing industries.
}

\author{
SANTOFIMIA, M.J., VILLANUEVA, F.J., CABA, J., FERNANDEZ-BERMEJO, \\ J., DEL TORO, X., WIRATUNGA, N., TRAPERO, J.R., RUBIO, A., \\ SALVADORI, C. and LOPEZ, J.C.
}

(C) 2021 IEEE. Personal use of this material is permitted. Permission from IEEE must be obtained for all other uses, in any current or future media, including reprinting/republishing this material for advertising or promotional purposes, creating new collective works, for resale or redistribution to servers or lists, or reuse of any copyrighted component of this work in other works. 


\title{
Autonomous CPSoS for Cognitive Large Manufacturing Industries
}

\author{
Maria Jose Santofimia*, Felix J. Villanueva*, Julian Caba*, Jesus Fernandez-Bermejo*, Xavier del Toro*, \\ Nirmalie Wiratunga ${ }^{\dagger}$, Juan R. Trapero ${ }^{\ddagger}$, Ana Rubio*, Claudio Salvadori ${ }^{\S}$ and Juan Carlos Lopez \\ ${ }^{*}$ Institute of Technology and Information Systems, University of Castilla-La Mancha, Ciudad Real, Spain. \\ ${ }^{\dagger}$ School of Computing, Robert Gordon University, Aberdeen, UK. \\ $\ddagger$ Department of Business Administration. University of Castilla-La Mancha, Spain. \\ $\S$ New Generation Sensors srl. Pisa, Italy. \\ Email: \{mariajose.santofimia, felix.villanueva, julian.caba, jesus.fruiz, xavier.deltoro, juanramon.trapero, \\ ana.rubio,juancarlos.lopez\}@uclm.es,n.wiratunga@rgu.ac.uk, claudio.salvadori@ngs-sensors.it
}

\begin{abstract}
The general aim of a cognitive Cyber Physical System of Systems (CPSoS) is to provide managed access to data in a smart fashion such that sensing and actuation capabilities are connected. Whilst there is significant funding and research devoted to this area, focus remains purely on creating bespoke systems. This paper presents a novel approach, based on a set of components to leverage Situational Awareness and Smart Actuation in large manufacturing industries with the focus on enabling predictive maintenance for asset and abnormal situation management. This paper presents a novel generic platform, named AtiCoS, that combines case-based and common-sense reasoning, as the enabling methodologies for enhancing CPSoS with cognitive capabilities.
\end{abstract}

Index Terms-CPSoS, Predictive Maintenance, Situational Awareness, Smart Actuation.

\section{INTRODUCTION}

$\mathbf{S}$ EVERAL market data providers agree on a positive outlook of the industry on the medium term. According to MarketsandMarkets [1] the predictive maintenance market will grow from $\$ 4.0$ billion in 2020 to $\$ 12.3$ billion by 2025 , and will exhibit a rising trend of Compound Annual Growth Rate (CAGR) up to the $25.2 \%$ for the forecast period. More optimistic predictions have been made by Statista.com [2] for an average CAGR of almost 40\% between 2018 and 2024.

Despite such positive market outlook, several business needs are still unmet by the current technology. According to a survey sponsored by GE [3], an extremely high percentage of European manufacturing industries are still unsatisfied with existing maintenance processes. A staggering $73 \%$ of respondents still performs maintenance with human inspectors using instrument readouts, whereas only $25 \%$ of the sample uses real-time monitoring techniques. This highlights the large gap for improvement in IoT predictive maintenance deployment and technology. In this market, CPSoS are an essential enabling technology. In the research and innovation agenda for the years 2016-2025 on cyber-physical systems of systems elaborated by the European Project CPSoS [4], predictive maintenance is listed as one of the 11 priorities in the mediumterm. This same agenda identifies three core challenges: 1) cognitive CPSoS; 2) distributed, reliable and efficient man- agement of CPSoS; and 3) the need for engineering support for the design-operation continuum of CPSoS.

From a software perspective, NESSI, the Horizon 2020 European Technology Platform (ETP) for software, services and data published a white paper identifying the challenges and opportunities for future CPS [5]. Current engineering principles are not appropriate for systems that, like CPSoS, cannot assume a closed world with predefined and prescribed interactions. CPSoS has to be constructed in an evolutionary and dynamic manner. This work proposes a disruptive AIbased approach that combines common-sense and case-based reasoning to dynamically evolve systems on run-time. Machine Learning (ML) algorithms are also proposed to support Situational Awareness and Smart Actuation processes.

Predictive analytics is proposed to improve predictive maintenance performance. Essentially, predictive maintenance relies on condition-based monitoring (CBM), where a key variable (temperature, vibration, etc.) is monitored and only when a threshold value is exceeded, the maintenance operation is launched. However, CBM does not really predict the state of health of the asset, i.e., CBM does not provide prognosis information. The architecture proposed in AtiCoS resorts to a set of predictive analytic tools ranging from time series analysis to machine learning algorithms to provide a real prediction of the remaining life time of the components based on all the digital information acquired. These predictions will be updated in real time to help scheduling the maintenance operation with a lower cost. Furthermore, AtiCoS relies on case-base and common-sense reasoning techniques to comprehensively tackle the challenge of understanding the situation that is taking place in the manufacturing facility (by interpreting the sensed events) and by proposing appropriate responses that extends those available in predefined plans. Reasoning about the situation makes use of both the common-sense knowledge and the experiential knowledge in the casebase of past similar situations. Run-time responses are enabled by a novel mechanism that support code injection, in a self-driven manner, based on the system knowledge (machine learning models, case-base and common-sense knowledge). In this sense, the main contributions of this paper can be summarized as follows: 
- AtiCoS proposes a groundbreaking architecture for predictive maintenance for large manufacturing facilities through disruptive Artificial Intelligence technologies that combine case-based and common-sense reasoning with machine learning approaches.

- AtiCoS supports comprehensive dataflows for CPSoS from the Edge through the Fog to the Cloud, which eventually contribute to more trustworthy and secure systems.

This paper is organized as follows. In Section II we present the background to this work followed by the proposed architecture, AtiCoS, in Section III. This architecture supports Situational Awareness and Smart Actuation features, so the next section describes how these functionalities are supported. Finally, the main conclusions of this work are summarized.

\section{BACKGROUND}

Industrial maintenance can be divided in to three main strategies [6]: i) Corrective maintenance, also known as failure-based maintenance. In this strategy, units are either replaced or repaired after a failure occurs. Note that maintenance is delayed if no spare part is available which may induce high down-times; ii) Preventive maintenance strategy performs actions to retain an item in a specified condition while the system is operating; iii) Predictive maintenance also called condition-based maintenance is based on the periodical acquisition of data to observe the state of the system. The parts are replaced when the observations reach a determined threshold value beyond which normal system operation is jeopardized. Most of the published bibliography consider the implementation of condition-based maintenance by monitoring the current level of degradation, however, they do not predict the future degradation. Note that, instead of assuming a statistical distribution (typically Weibull), AtiCoS builds empirical distributions that will be updated with new actual observations from sensors that will yield real-time predictions of the remaining life of the analysed elements. This novel point of view provides a data-driven predictive maintenance methodology. Another novelty of the proposed methodology is to incorporate energy efficiency indicators to help detect potential failures whenever the energy consumption of components is increased with respect to the normal (nonfault) consumption. Note that this loss is generally ignored by both academics and practitioners alike [7].

\section{ThE PROPOSED ARCHITECTURE: AtiCoS}

\section{A. Principles inspiring the AtiCoS architecture}

AtiCoS is aimed at supporting predictive maintenance for improved asset management and abnormal situation management, in the context of large manufacturing facilities. Two dimensions of predictive maintenance are considered in AtiCoS: the "known unknowns" and the "unknown unknowns" [8]. The term "known unknowns" is used to refer to those situations for which software systems have a predefined set of adaptation strategies. On the contrary, the term "unknown unknowns" refers to those situations for which there is no predefined set of adaptations and therefore, cannot be conveniently addressed. The challenge, as stated by the NESSI [8], is "to identify new strategies to adapt systems to new and emerging requirements, unforeseen interactions with other systems or new and changing contexts", all that at run-time. AtiCoS considers different approaches for handling both the "known unknowns" and "unknown unknowns". The known ones will be addressed through advanced and novel techniques for Big Data Analytics and Machine Learning approaches, and CaseBased Reasoning. For the unknown ones a Common-Sense Reasoning approach will be employed to provide foundational knowledge of how the world (in general) and certain industrial processes (in particular) work.

Additionally, AtiCoS builds around the idea of "think globally act locally" as depicted in Figure 1. To think globally the considered architecture should have access to the information generated at the different dimensions (systems) of an industrial process (Situational Awareness). To act locally, apart from determining the most appropriate response, it is necessary to identify how those responses are to be implemented (Smart Actuation).

\section{B. The three-computational models of AtiCoS}

At edge level, both real and virtual (digital twins) devices will be running. These devices will be employed to monitor and support actuation over the industrial systems. The Cognitive Operator Console, at the fog level, is the interface between these devices and the human operators. Operators will actuate through this console, commanding instructions to either real or virtual equipment, in a transparent manner for the operator. Similarly, operators will obtain information from sensors and equipment either real or virtual, also in a transparent manner. This console will also provide access to historical data stored in the cloud as well as predictions, the general state of the plant or specific elements of it.

Data as provided by sensors have to be pre-processed for feature extraction and dimensionality reduction and pattern recognition. To this end, FPGA-based nodes will perform the first computational works. Data will also be translated into a qualitative feature, normally based on thresholds. The data preprocessing module will therefore provide two measures: one considered quantitative and another one considered qualitative. The quantitative one will be the input for the machine learning algorithms running at the fog. Trained models obtained from the training stages run at the cloud will be deployed in FPGAs nodes. A first prediction of the useful-lifetime or situation will be carried out. Based on the obtained result (either within normality or not) different paths will be enabled in the Situational Awareness and Smart Actuation components.

Two elements comprise the Situational Awareness component. On the one hand, the Machine Learning (ML) component will consider classification algorithms for predicting which component is going to fail and when. If evidence is strong then the Situational Awareness component will trust such a prediction and consider it the single hypothesis. Thereafter the Solution Proposer will look to the case-base for a match. If there is a match (i.e. this is a "known unknown") the associated 


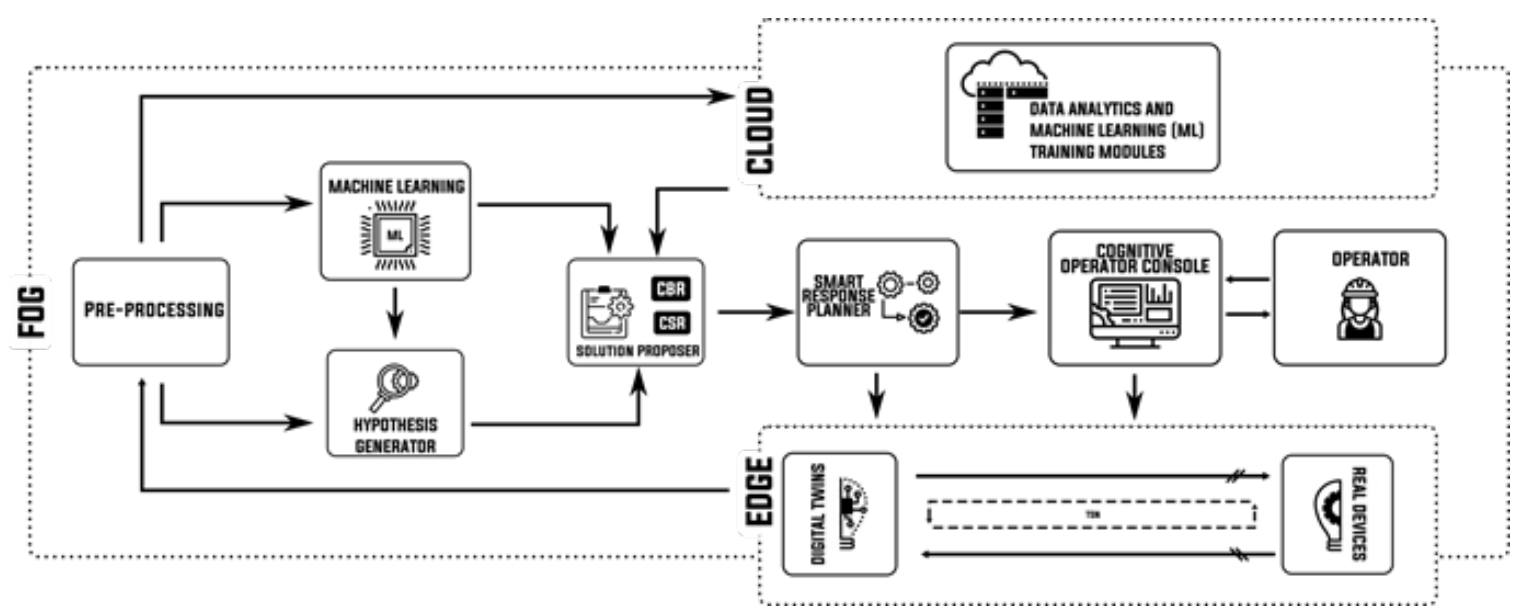

Fig. 1. Overview of the AtiCoS dataflow

plan will be provided to the Smart Response Planner component, which will eventually devise the most appropriate way to undertake it. These actions will be autonomously executed because a good match was found in the case-base. The solution will be displayed in the Cognitive Operator Console.

Alternatively, if the Machine Learning algorithm yields weak evidence, it is the role of the Common-Sense Reasoning (CSR) component either to provide confirmation or to propose an alternative interpretation. Following an episodic-reasoning approach [9], qualitative measures are considered, in parallel, as hypothesis of different ongoing situations. Whenever one hypothesis stands out over the others, it is considered the most plausible causal explanation. If the case-base has a match for that situation the solution is applied. On the contrary, if there is no good match among the existing cases, the CommonSense Reasoning component will devise the most appropriate solution based on the general and domain specific knowledge captured in the knowledge base. The proposed solution will be provided to the Smart Response Planner (SRP) which will, eventually, adapt existing code to run the proposed solution. Successful solutions are retained for future reuse in the casebase, thereby improving the experiences of the planner

\section{Situational Awareness And Smart ACtuation}

AtiCoS proposes a five-stage approach (see Figure 2) for understanding ongoing situations based on the information provided by sensors and devising appropriate ways to handle them.

\section{A. The Pre-Processing Module}

Situational awareness is very challenging because of the heterogeneity, uncertainty, and imprecision of the data used for situation interpretation. During data collection different sources of information are employed, therefore involving different devices, technologies, protocols, etc., turning data homogenisation is yet another major challenge. Data homogenisation is necessary before any further analysis and this task is carried out by the Pre-Processing module.

\section{B. The Machine Learning Module}

Once homogenized, the next step for Situational Awareness consists in a first classification of the data. The Machine Learning component will evaluate and select the most appropriate algorithm, among the different ones suitable for this end. This component will yield both the classification and its confidence score. Depending on that, as well as the accuracy of the models and algorithms, this first classification will be assigned a level of credibility. When credible (i.e.: accuracy is over a certain threshold), the recognized situation will be directly provided to the Solution Proposer module (SP) which will devise the most appropriate means to handle it. However, if the evidence is not strong enough for that interpretation of the ongoing situation the Hypothesis Generator module will come into play.

\section{The Hypothesis Generator Module}

AtiCoS conceives the Hypothesis Generator (HG) module as the component responsible for seeking causal explanations for sensed events. According to Woodaward [10] a causal explanation is "any explanation that proceeds by showing how an outcome depends (where the dependence in question is not logical or conceptual) on other variables or factors counts as causal". For example, an explanation for a big crash noise and a sudden stop of a line can be the occurrence of an explosion. Explanations should be considered as preliminary hypothesis since there might be many different explanations catering for the same sensorial information, and it cannot be obviated that events are neither always clear nor precise due to sensors malfunctioning or low precision. For that reason, inspired by work in [11] we propose a seven-stage process for hypothesis generation (see Figure 3).

A first stage will be intended to associate sensor measures (as captured effects of events) to the actions that might have caused them. This view has been identified as the dT point of view. Then, a second stage will take care of identifying the situation in which such sequence of actions could make sense. This view has been referred as $\Delta \mathrm{T}$ point of view. Finally, the $\mathrm{T}$ domain point of view will be accomplished by the big data analytics. Combining the $\mathrm{dT}$ and $\Delta \mathrm{T}$ point of views will bring 


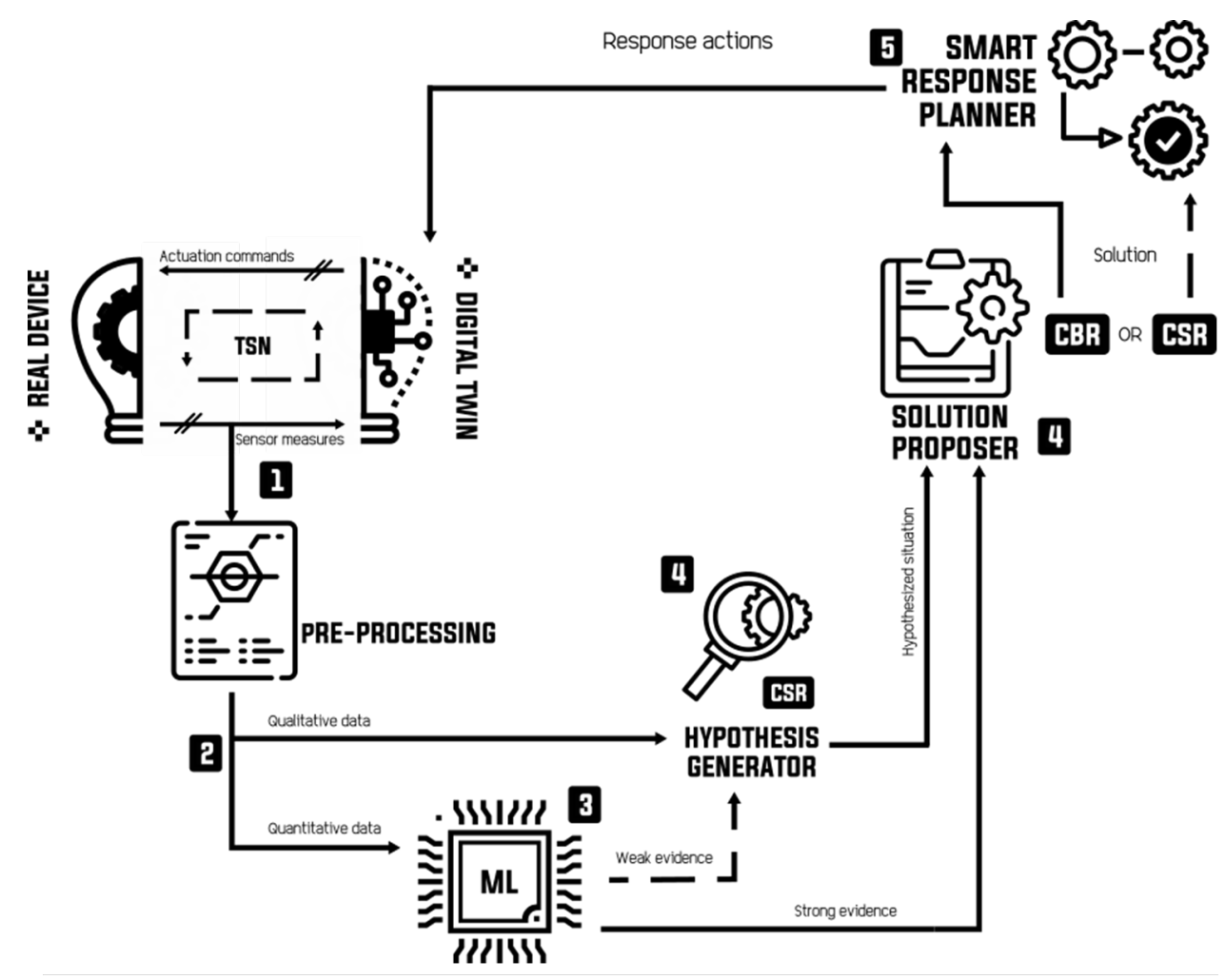

Fig. 2. Stages for Situational Awareness and Smart Actuation

into light inconsistent interpretations or, alternatively, confirm the action initially hypothesized.

Based on the theory of situation calculus, proposed by McCarthy and Hayes [12] to formally represent world changes as situations, we plan to extend it with the possible world theory [13]. In this sense, the possible world abstraction is going to be employed to simultaneously consider parallel, and therefore inconsistent, causal explanations until enough information is available to support the election of one hypothesis over the others. Each world is plausible within itself although incongruous among the others. Eventually, only one hypothesis will be considered plausible and this will be provided to the Solution Proposer module to work out the best way to address it.

\section{The Solution Proposer Module}

This module combines Case-Based and Common-Sense Reasoning techniques.

The hypothesis of the ongoing situation yielded by either the Machine Learning or the Hypothesis Generator component is considered the situational description provided as input for the Solution Proposer. This module will compare that situational description to all known situational instances in the case-base and, when a match is detected, it will return a response that is adapted for the current query. The development of the casebased reasoning component consists of the following main steps:

1) Retrieval of similar cases: Implementation of the case comparison using existing libraries and tools such as COLIBRI [14] Studio or CloodCBR [15]
2) Reuse the solution from the case-base: The first stage is to use local and global similarity metrics to compare a query case with stored situational awareness cases in the case base. This typically involves a pair-wise similarity computation to compare cases.

3) Revise the solution from the case-base: Once a matching case is found, the case-based reasoning component has to refine the solution to fit the current situation by taking into account the differences between the current situation and the differences between the best matching case. For instance the type of asset may be more specialised and the compliance constraints will need to be considered. This may add further steps in to the maintenance plan, which in turn will impact the maintenance prediction. Revision will be guided by the commonsense reasoning component which will be based on the differences between the situational descriptors and any conflicts will be resolved accordingly. Output of this stage is an adapted maintenance plan for the queried situation

4) Retention of cases: Once the system has created a new problem/solution pair that is sufficiently dissimilar to existing cases, common-sense reasoning will be triggered with regards to case retention policies. These heuristics will guide the level of novelty that is required in a new refined solution before it can be added as case in its own right within the case-base. Once the decision is made to retain the case then the necessary situational descriptors together with the lessons learned and explanations represented and the case added to the 


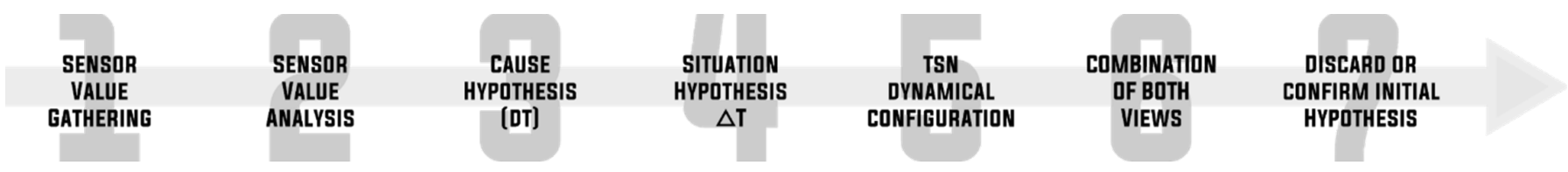

Fig. 3. Stages for hypothesis generation

existing case-base, thereby creating the feedback loop of the experiential learning cycle

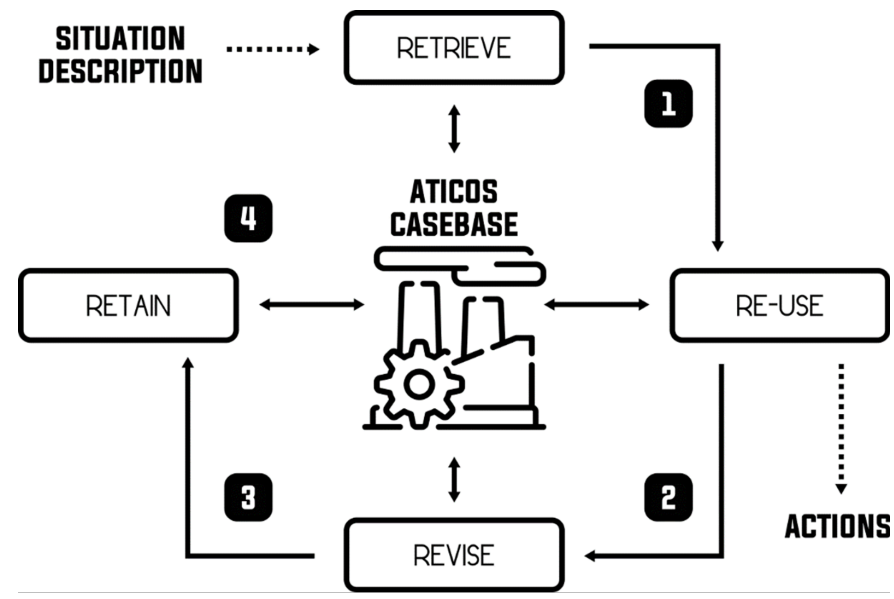

Fig. 4. The feedback loop of the experiential learning cycle

As summarized in Figure 4, the idea is to determine whether there is a match in the case-base for that same situation. If so, the solution will be reused by devising (this is the goal provided to the Smart Response Planer) the set of actions that will be executed. If there is no exact match, commonsense knowledge can be used to generalise concepts so as to improve the retrieval of relevant similar cases using analogical reasoning. Heuristics can be used to check which requirements are not being met and then, using common-sense knowledge to adapt the existing solution by reasoning about the differences between the actual and the retrieved situation descriptions. Finally, common-sense knowledge will also be used to explain why this is a good solution and store it in the case-base as a new experience.

\section{E. The Smart Response Planner Module}

The Solution Proposer will yield a solution (stage 5 of Figure 3) to the Smart Response Planner. Two challenges are faced here: how should system responses be driven by the CPSoS and how are they going to be articulated. Regarding the first aspect, AtiCoS plans to provide a model that describes the propositional attitudes that are going to guide its behavior. The propositional attitudes or mental states will capture the system knowledge, the system objectives, and the available means. This knowledge will be obtained from interviewing with operators. Regarding the support to automatic and unsupervised system responses, the first challenge to be faced is how to address a context in which available means or systems cannot be foreseen in advance. Then, predefined system responses cannot be elaborated in terms of available system or services because they might or might not be available at that moment. Establishing a similarity with a common situation faced by people in our daily life, during a blackout or while in the dark, we look for sources of light at hand, being our mobile phone the most common solution. This is not pre-coded as we have the ability to devise clever uses of things we have at hand. Basically, this capability is based on the well understanding we have about how things works. This enables us to derive the phone's capability to work as a torch or source of light.

In the context of CPSoS, the only way of figuring out an alternative way of accomplishing a task for which there is not any specific system is by composing or reconfiguring available ones into a new system whose combine functionality emulates the desired one. This process has to be undertaken in an autonomous and self-sufficient way without requesting the user or programmer intervention.

AtiCoS resort to the a semantic composition approach [16] based on the profound understanding of the capabilities, held at this level, by hardware devices and systems. This analytical capability will be driven by a planning scheme that, given a desired result (illuminate a space) it is going to look for a reconfiguration or composition setup through the whole space of available resources. To enable such a setup, a common semantic model should be shared among the different layers of the architecture. Finally, it has to be highlighted that the strength of the planning scheme resides at the knowledge it holds about how the world works, also known as common sense, and the detailed descriptions of hardware devices, services, and systems: what they are, how the work, what they are capable of, etc. We plan to use Scone ${ }^{1}$ to model and hold AtiCoS knowledge. Scone is also particularly well suited to manage possible worlds, through its implementation of the multiple-contexts mechanism [17]. Eventually, the Smart Response Planner will articulate the execution of the solution plan by interacting directly over systems (through the digital twins). An element known as the Wiring Service (a solution made available by [16]) will take care of supporting code injection in runtime to make possible the execution of the plan.

\section{CONCLUSION}

AtiCoS plans to propose a reference architecture with systems and components that support CPSoS designers towards autonomous and smart CPSoS. To this end, the project architecture ambitiously aims to support the construction of systems that, based on a myriad of technologies, are capable of understanding events (Situational Awareness) and evolving systems at run-time without a previously dictated plan (Smart

\footnotetext{
${ }^{1}$ http://www.cs.cmu.edu/ sef/scone/
} 
Actuation). AtiCoS will address the challenge of enhancing CPSoS with cognitive capabilities what will, eventually, lead to enable distributed, reliable and efficient management of such systems. To demonstrate the achievement of this general goal, AtiCoS focuses on enabling predictive maintenance for improved asset and abnormal situation management resorting to different Artificial Intelligence techniques. Two types of situations will be considered to show the need for two levels of intelligence. Machine learning techniques will be employed to learn behavioral patterns, trends, and models that help the reasoning system to recognise the known unknowns from experiential knowledge. More sophisticated reasoning mechanisms are required, rather than just classification or characterization tasks, in order to address (in an autonomous and trustworthy manner) the unknown unknowns. A common-sense-based approach will be employed to address this situations and appropriate resources will be made available to speed up the process of knowledge gathering and modelling.

\section{ACKNOWLEDGMENT}

This paper has been funded by European Union's Horizon 2020 research and innovation programme under grant agreement no. 857159, project SHAPES (Smart \& Healthy Ageing through People Engaging in Supportive Systems), the Ministry of Economy and Competitiveness (MINECO) of the Spanish Government (TALENT-BELIEF project, no. PID2020-116417RB-C44), by Spanish Ministry of Education, Culture and Sport within the framework of the National FPU Program (ref. FPU FPU19/03387), and the Regional Government of Castilla-La Mancha under FEDER funding (SymbIoT project, no. SBPLY-17-180501-000334).

\section{REFERENCES}

[1] S. Ray, "Implication of predictive maintenance for industrial marketing: A case study from the air compressor industry," in Innovations in the Industrial Internet of Things (IIoT) and Smart Factory. IGI Global, 2021, pp. 231-243.

[2] S. R. Department, "Worldwide - predictive maintenance market size 2024," Feb 2021. [Online]. Available: \url\{https://www.statista.com/ statistics/748080/global-predictive-maintenance-market-size/ $\}$

[3] M. Milojevic and F. Nassah, 2018. [Online]. Available: url\{https://www.plm.automation.siemens.com/media/global/en/ PAC $\backslash$ PPredictive $\backslash$ Maintenance $\backslash$ SSiemens $\backslash$ Executive $\backslash$ Summary $\backslash$ _ 2018-71130 \tcm27-33237.pdf $\}$

[4] S. Engell, R. Paulen, C. Sonntag, H. Thompson, M. Reniers, S. Klessova, and B. Copigneaux, "Proposal of a european research and innovation agenda on cyber-physical systems of systems 2016-2025," CPSoS Project, TU Dortmund, 2016.

[5] A. Metzger et al., "Cyber physical systems: Opportunities and challenges for software, services, cloud and data," NESSI white paper, 2015.

[6] A. Van Horenbeek, J. Buré, D. Cattrysse, L. Pintelon, and P. Vansteenwegen, "Joint maintenance and inventory optimization systems: A review," International Journal of Production Economics, vol. 143, no. 2, pp. 499-508, 2013, focusing on Inventories: Research and Applications. [Online]. Available: https://www.sciencedirect.com/ science/article/pii/S0925527312001466

[7] G. Singh, T. Anil Kumar, and V. Naikan, "Efficiency monitoring as a strategy for cost effective maintenance of induction motors for minimizing carbon emission and energy consumption," Reliability Engineering \& System Safety, vol. 184, pp. 193-201, 2019, impact of Prognostics and Health Management in Systems Reliability and Maintenance Planning. [Online]. Available: https://www.sciencedirect. com/science/article/pii/S0951832017310979

[8] N. E. Software and S. Initiative, "Software Continuum. Recommendations for ICT Work Programme 2018+," NESSI white paper, 2016.
[9] M. J. Santofimia, J. Martinez-del Rincon, and J.-C. Nebel, "Episodic reasoning for vision-based human action recognition," The Scientific World Journal, vol. 2014, 2014.

[10] J. Woodward, Making things happen: A theory of causal explanation. Oxford university press, 2005.

[11] F. Galatioto, C. Salvadori, M. Petracca, J. G. Herrero, M. Santofimia, and A. Pollini, "Smart cyber systems incorporating human-in-the-loop towards ergonomic and sustainable transport systems," in Transport Infrastructure and Systems. CRC Press, 2017, pp. 875-882.

[12] J. McCarthy and P. J. Hayes, "Some philosophical problems from the standpoint of artificial intelligence," in Readings in artificial intelligence. Elsevier, 1981, pp. 431-450.

[13] P. Forrest, "Possible worlds," 2004

[14] J. A. Recio-Garcia, B. Diaz-Agudo, and P. A. González-Calero, Successful Case-based Reasoning Applications-2, ser. Studies in Computational Intelligence. Springer Berlin Heidelberg, 2014, vol. 494, inbook The COLIBRI Platform: Tools, Features and Working Examples, pp. 55-85.

[15] I. Nkisi-Orji, N. Wiratunga, C. Palihawadana, J. A. Recio-García, and D. Corsar, "Clood cbr: Towards microservices oriented case-based reasoning," in Case-Based Reasoning Research and Development, I. Watson and R. Weber, Eds. Cham: Springer International Publishing, 2020, pp. 129-143.

[16] M. J. Santofimia, D. Villa, O. Aceña, X. del Toro, C. Trapero, F. J. Villanueva, and J. C. Lopez, "Enabling smart behavior through automatic service composition for internet of things-based smart homes," International Journal of Distributed Sensor Networks, vol. 14, no. 8, p. 1550147718794616, 2018.

[17] M. J. Santofimia, S. E. Fahlman, X. del Toro, F. Moya, and J. C. Lopez, "Possible-world and multiple-context semantics for common-sense action planning," in Situational Awareness for Assistive Technologies. IOS Press, 2012, pp. 9-49. 